\title{
The effect of electromagnetic guidance system on early learning curve of ultrasound for novices
}

\author{
Eun Jung Kim ${ }^{1}$, Jiyoung Min ${ }^{1}$, Jeongyun Song ${ }^{2}$, Kyungchul Song ${ }^{2}$, \\ Jang-Ho Song ${ }^{2}$, and Hyo Jin Byon ${ }^{1}$ \\ Department of Anesthesiology and Pain Medicine, ${ }^{1}$ Anesthesia and Pain Research Institute, Yonsei University \\ College of Medicine, Seoul, ${ }^{2}$ Inha University College of Medicine, Incheon, Korea
}

\begin{abstract}
Background: Electromagnetic guidance reveals needle alignment and position relative to the image plane on an ultrasound view. This study compared the early learning curves of novices performing ultrasound-guided needle placement with $(n=10)$ or without electromagnetic guidance $(n=10)$.

Methods: Participants performed 30 ultrasound-guided needle placements using an echogenic stick (0.3-cm diameter) as a target inside a phantom model; this early learning period was divided into sequential periods (P1: 1-5, P2: 6-10, P3: 11-15, P4: 16-20, P5: 21-25, P6: 26-30 attempts).

Results: Using an in-plane approach, the time required for needle placement in the EMG group was significantly shorter than that of the non-EMG group in P1, P2, P4, and P6 and the number of needle advances of the EMG group was significantly smaller than that of the non-EMG group in P1 and P2. Using an out-of-plane approaches, the time required for needle placement in the EMG group was significantly shorter than that of the non-EMG group in all periods, but the number of needle advances was similar between both groups in P1-P5.

Conclusions: The electromagnetic guidance system may be beneficial when performing ultrasound guided peripheral nerve blocks or vascular cannulation in the early learning period, especially by inexperienced operators with reducing patient risk.
\end{abstract}

Key Words: Electromagnetic fields, Learning curve, Ultrasonography.

Corresponding author: Hyo Jin Byon, M.D., Ph.D.

Department of Anesthesiology and Pain Medicine, Anesthesia and Pain Research Institute, Yonsei University College of Medicine, 50-1 Yonsei-ro, Seodaemun-gu, Seoul 120-752, Korea

Tel: 82-2-2227-4641, Fax: 82-2-2227-7897

E-mail: jinoben@yuhs.ac

ORCID: http://orcid.org/0000-0002-5400-6155

Received: June 10, 2015.

Revised: 1st, July 21, 2015; 2nd, August 10, 2015.

Accepted: August 12, 2015.

Korean J Anesthesiol 2016 February 69(1): 15-20

http://dx.doi.org/10.4097/kjae.2016.69.1.15

\section{Introduction}

An ultrasound guidance for procedures such as central venous catheter insertion and nerve block allows direct visualization of anatomical structure and needle placement, which may increase success rate and decrease complications [1]. However, operators vary in their ability to acquire and interpret ultrasound images, needle-beam alignment, and real-time tracking of the needle trajectory for ultrasound-guided needle placement [2-6]. Novices may have difficulty adjusting needle direction while keeping an eye on the screen, resulting in injury to surrounding tissue and vascular structures $[7,8]$. The eZono ${ }^{\mathrm{TM}} 4000$ portable ultrasound device (eZono AG, Jena, Germany) is equipped with an electromagnetic guidance system (eZGuide ${ }^{\mathrm{TM}}$ ) that shows the alignment and position of the needle relative to the image

(c) This is an open-access article distributed under the terms of the Creative Commons Attribution Non-Commercial License (http://creativecommons.org/ licenses/by-nc/4.0/), which permits unrestricted non-commercial use, distribution, and reproduction in any medium, provided the original work is properly cited. 
plane on the ultrasound screen. This system allows the operator to identify the correct needle trajectory prior to puncturing the skin and maintain the chosen route to the target anatomy, which could help novices learn the ultrasound-guided needle placement technique. The aim of this study was to compare early learning curves of novices performing ultrasound-guided needle placement in a phantom model with or without electromagnetic guidance.

\section{Materials and Methods}

After the study protocol was approved by the Hospital Institutional Review Board, 20 anesthesiology residents and interns were prospectively enrolled. All participants were provided written informed consent for participation in the study. The participants, who had no prior experience with ultrasoundguided needle placement, were randomly allocated into two groups using Research Randomizer ${ }^{\mathrm{TM}}$ (http://www.randomizer. org) : participants who performed ultrasound-guided needle placement with electromagnetic guidance (EMG group) versus without electromagnetic guidance (non-EMG group). All participants received basic instruction on ultrasound device usage, and in-plane and out-of-plane approaches. Participants in the EMG group received additional instruction regarding the use of an electromagnetic guidance system with a Stimuplex ${ }^{\circledR}$ needle (22 Gauge $\times 50 \mathrm{~mm}$ insulated needle, $30^{\circ}$ bevel; B. Braun, Bethlehem, PA, USA) [9], whereas those in the non-EMG group watched a demonstration of conventional ultrasonography. The phantom (article number 6010001; eZono AG, Jena, Germany) was $6 \times 12 \times 3 \mathrm{~cm}$ (height $\times$ width $\times$ depth), and an echogenic stick (0.3-cm diameter) was embedded as a target $1.5 \mathrm{~cm}$ under the surface. We used the eZono ${ }^{\mathrm{TM}} 4000$ device (eZono AG, Jena, Germany) equipped with eZGuide ${ }^{\mathrm{TM}}$ electromagnetic guidance system and linear probe (L3-12NGS, eZono AG, Jena, Germany). After ultrasound gel was applied on the phantom, all participants were able to obtain an optimal view of the ultrasound image, which is defined as follows. For the in-plane approach, the target appears as a straight tube across the screen (longaxis view). For the out-of-plane approach, the target appears as a $3-\mathrm{mm}$ diameter circle in the middle of the screen (short-axis view).

While maintaining an optimal view, the participants in the non-EMG group inserted the needle into the phantom and advanced the needle toward the target using conventional ultrasonography. For the in-plane approach, the participants advanced the needle parallel to the long axis of the probe. For the out-of-plane approach, the participants advanced the needle perpendicular to the long axis of the probe. When the needle touched the echogenic stick, observers confirmed that needle placement was a success. If the needle did not touch the target, the participant removed the needle from the phantom and made another attempt. In the EMG group, participants performed the procedure using a needle that had been passed through a magnetic field. As the magnetized needle approached the probe, the screen showed its position relative to the probe, the expected route of the needle, and the needle tip position. For the in-plane approach, participants advanced the needle parallel to the long axis of the probe. When the needle tip was located in the middle of the short axis, the needle at the left upper screen changed from red to green. The needle trajectory appeared as a dotted line, and the actual position of the needle was shown between two solid lines. When the needle tip was placed in the ultrasound image plane, the needle tip appeared as a square box (Fig. 1). The operator advanced the needle toward the target until the two solid lines, regarding as the actual needle position, reach the target indicating the contact of the needle tip with the target. For the out-of-plane approach, participants advanced the needle perpendicular to the long axis of the probe. When the needle tip was located in the middle of the long axis, the needle shown in the left upper screen changed from red to green. As in the inplane approach, the needle trajectory appeared as a dotted line, the needle position was shown between two solid lines, and the needle tip appeared as a square box in the image plane (Fig. 2). The operator advanced the needle toward the target until the two solid lines met the target. If the operator could not touch the target with the needle, the needle was removed from the phantom, and the procedure was attempted again.

All participants attempted five in-plane approaches and five

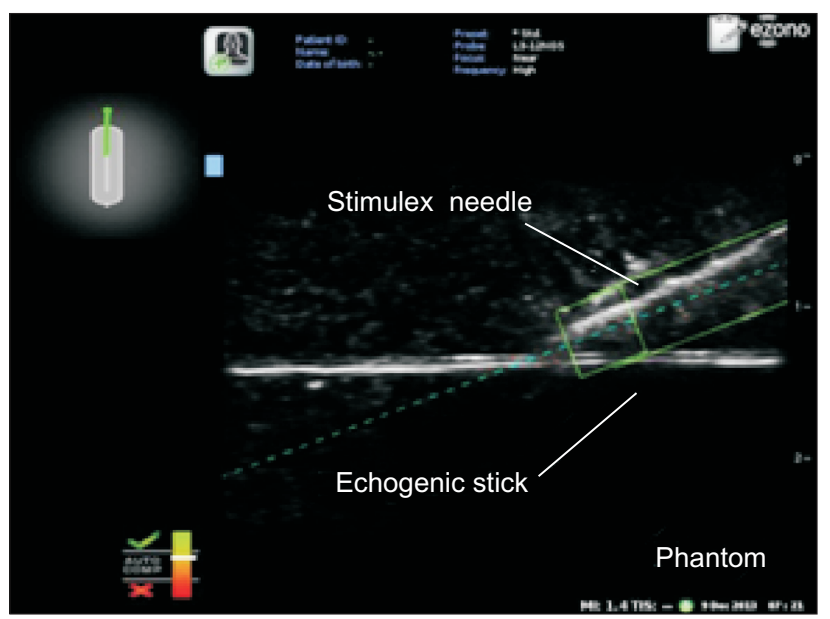

Fig. 1. Ultrasound image with electromagnetic guidance in the in-plane view. The needle trajectory is shown as a dotted line, the needle position is shown between the two solid lines, and the needle tip appears as a square box. The diagram of the needle in the left upper screen changes from red to green when the needle tip is in the middle of the short axis of the probe. The needle (Stimuplex ${ }^{\circledR}$ ) was advanced toward a $0.3-\mathrm{cm}$ diameter echogenic stick embedded $1.5 \mathrm{~cm}$ under the surface of the phantom model. 


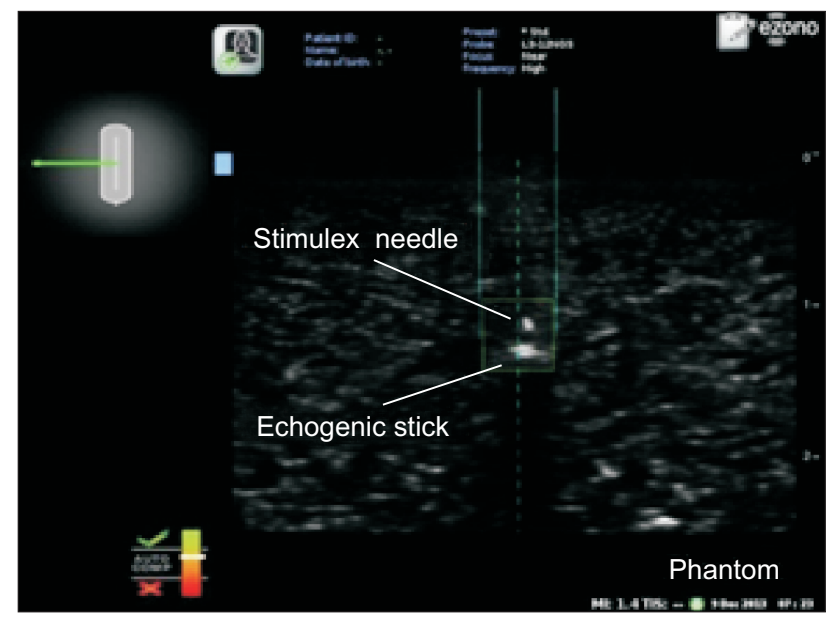

Fig. 2. Ultrasound image with electromagnetic guidance in the out-ofplane view. The needle trajectory is shown as a dotted line, the needle position is shown between the two solid lines, and the needle tip appears as a square box. The diagram of the needle in the left upper screen changes from red to green when the needle tip is in the middle of the long axis of the probe. The needle (Stimuplex ${ }^{\circledR}$ ) was advanced toward a $0.3-\mathrm{cm}$ diameter echogenic stick embedded $1.5 \mathrm{~cm}$ under the surface of the phantom model.

out-of-plane approaches each day for six days. Any participant who performed ultrasound-guided needle placements during this time other than those related to this study was eliminated from the study. Participants were not allowed to observe each other performing this procedure during the experimental period. During each attempt, two observers recorded the score for the ultrasound view, number of needle advances required for reaching the target, and time required for needle placement (defined as time elapsed between placing the needle in the phantom model and touching the target).

The ultrasound view for the in-plane approach was given a score of 0 to 3 as follows: 0 , complete needle visualization during its insertion; 1 , the needle tip was visible during the entire procedure, but the shaft was only partially visible; 2 , only the needle tip was visible during the attempt; or 3 , only phantom distortion was observed. The ultrasound view for the out-of-plane approach was given a score of 0 to 2 as follows: 0 , needle tip contacting the target was visible; 1 , when the needle tip contacted the target, part of the needle shaft was visible but not the needle tip; or 2, when the needle tip contacted the target, only distortion of the phantom was observed. The first 30 attempts $[10,11]$ for each approach (in-plane and out-of-plane) were divided into six sequential periods (P1: 1-5, P2: 6-10, P3: 11-15, P4: 16-20, P5: 21-25, P6: 26-30). The time required for needle placement, the number of needle advances required for reaching the target, and score for the ultrasound view for each time period were compared between the two groups (EMG vs. non-EMG).
Table 1. Demographic and Clinical Characteristics of Participants

\begin{tabular}{llccc}
\hline & & $\begin{array}{c}\text { Non-EMG } \\
(\mathrm{n}=10)\end{array}$ & $\begin{array}{c}\text { EMG } \\
(\mathrm{n}=10)\end{array}$ & P value \\
\hline Sex & Male & $7(70)$ & $9(90)$ & 0.582 \\
& Female & $3(30)$ & $1(10)$ & \\
Training Grade & Intern & $5(50)$ & $4(40)$ & 1.000 \\
& Resident & $5(50)$ & $6(60)$ & \\
Age $(\mathrm{yr})$ & & $32.3 \pm 2.7$ & $31.1 \pm 2.3$ & 0.400 \\
\hline
\end{tabular}

Data are shown as numbers (\%) or mean \pm SD.

\section{Statistical analysis}

A pilot study with five participants for each group was performed prior to this study, and the time required for needle placement was analysed using repeated measures ANOVA. This design achieves $87 \%$ power to test factor B if a Geisser-Greenhouse Corrected F Test is used with a 5\% significance level and the actual effect standard deviation is 1.01 [12-14], generating an estimate of eight participants per group. Assuming a dropout rate of $20 \%$, we recruited 10 participants per group. Demographic data were compared using Fisher's exact test or Wilcoxon rank-sum test. The time required for needle placement and the number of needle advances were compared between groups by repeated measures ANOVA and Wilcoxon rank-sum test. The scores for the ultrasound view were compared using mixed models regression analysis and Wilcoxon rank-sum test. Statistical analyses were performed using SPSS version 19 (SPSS Inc, Chicago, IL, USA). Data are presented as mean $\pm \mathrm{SD}$, and $\mathrm{P}<0.05$ was considered significant.

\section{Results}

Demographic characteristics and the years of training were similar between two groups (Table 1). All participants performed 30 attempts at in-plane ultrasound-guided needle placement and 30 attempts at out-of plane ultrasound-guided needle placement within 6 days. Using the in-plane approach, a significant effect of electromagnetic guidance (group $\times$ time interaction) was observed regarding the time required for needle placement ( $\mathrm{P}=0.035$; repeated measures ANOVA), the number of needle advances required for reaching the target $(\mathrm{P}=0.037$; repeated measures ANOVA), and the score for the ultrasound view ( $\mathrm{P}=0.025$; mixed models regression analysis). The time required for needle placement in the EMG group was significantly shorter than that of the non-EMG group in the P1, P2, P4, and P6 periods. The number of needle advances was significantly less often in the EMG group compared with the non-EMG group in the P1 and P2 periods. In addition, the score for the ultrasound view was significantly higher in the EMG group than 
Table 2. Data for the In-plane Approach

\begin{tabular}{|c|c|c|c|c|c|c|c|c|c|}
\hline \multirow{2}{*}{$\frac{\text { In plane }}{\text { Period }}$} & \multicolumn{3}{|c|}{ Time for needle placement (Seconds) } & \multicolumn{3}{|c|}{ Number of needle advances (Numbers) } & \multicolumn{3}{|c|}{ Score for ultrasound view (Point) } \\
\hline & non-EMG & EMG & P value & non-EMG & EMG & $P$ value & non-EMG & EMG & $P$ value \\
\hline 1 & $17.84 \pm 11.34$ & $7.76 \pm 3.23$ & 0.037 & $3.44 \pm 1.83$ & $2.10 \pm 0.72$ & 0.045 & $2.14 \pm 0.35$ & $1.58 \pm 0.5$ & 0.018 \\
\hline 2 & $10.26 \pm 4.64$ & $5.80 \pm 1.98$ & 0.005 & $2.22 \pm 0.78$ & $1.48 \pm 0.50$ & 0.016 & $1.74 \pm 0.54$ & $1.22 \pm 0.26$ & 0.021 \\
\hline 3 & $7.22 \pm 3.57$ & $5.00 \pm 2.33$ & 0.121 & $1.48 \pm 0.62$ & $1.30 \pm 0.42$ & 0.404 & $2.10 \pm 0.58$ & $1.00 \pm 0.34$ & $<.001$ \\
\hline 4 & $4.90 \pm 2.22$ & $2.88 \pm 0.80$ & 0.037 & $1.14 \pm 0.21$ & $1.12 \pm 0.17$ & 0.932 & $2.00 \pm 0.57$ & $0.96 \pm 0.21$ & $<.001$ \\
\hline 5 & $5.24 \pm 2.13$ & $4.08 \pm 3.24$ & 0.064 & $1.18 \pm 0.20$ & $1.12 \pm 0.25$ & 0.239 & $2.04 \pm 0.30$ & $0.96 \pm 0.31$ & $<.001$ \\
\hline 6 & $4.86 \pm 1.76$ & $3.02 \pm 0.85$ & 0.017 & $1.08 \pm 0.14$ & $1.06 \pm 0.13$ & 0.654 & $1.98 \pm 0.27$ & $0.62 \pm 0.33$ & $<.001$ \\
\hline
\end{tabular}

Results are expressed as mean \pm SD. P values as compared two groups in each period.

Table 3. Data for the Out-of-plane Approach

\begin{tabular}{|c|c|c|c|c|c|c|c|c|c|}
\hline \multirow{2}{*}{$\frac{\text { Out of plane }}{\text { Period }}$} & \multicolumn{3}{|c|}{ Time for needle placement (Seconds) } & \multicolumn{3}{|c|}{ Number of needle advances (Numbers) } & \multicolumn{3}{|c|}{ Score for ultrasound view (Point) } \\
\hline & non-EMG & EMG & $\mathrm{P}$ value & non-EMG & EMG & P value & non-EMG & EMG & $\mathrm{P}$ value \\
\hline 1 & $8.14 \pm 5.51$ & $4.02 \pm 1.70$ & 0.012 & $2.10 \pm 1.17$ & $1.30 \pm 0.29$ & 0.092 & $1.40 \pm 0.23$ & $0.70 \pm 0.32$ & $<.001$ \\
\hline 2 & $5.74 \pm 3.39$ & $3.10 \pm 1.25$ & 0.021 & $1.58 \pm 0.75$ & $1.24 \pm 0.37$ & 0.293 & $1.26 \pm 0.40$ & $0.58 \pm 0.15$ & 0.001 \\
\hline 3 & $5.42 \pm 3.65$ & $2.66 \pm 1.12$ & 0.023 & $1.44 \pm 0.85$ & $1.02 \pm 0.06$ & 0.111 & $1.26 \pm 0.41$ & $0.72 \pm 0.19$ & 0.001 \\
\hline 4 & $4.38 \pm 2.79$ & $2.46 \pm 1.07$ & 0.04 & $1.18 \pm 0.37$ & $1.02 \pm 0.06$ & 0.121 & $1.28 \pm 0.27$ & $0.64 \pm 0.26$ & $<.001$ \\
\hline 5 & $4.18 \pm 2.08$ & $2.34 \pm 0.89$ & 0.03 & $1.20 \pm 0.25$ & $1.14 \pm 0.13$ & 0.839 & $1.56 \pm 0.21$ & $0.44 \pm 0.13$ & $<.001$ \\
\hline 6 & $3.80 \pm 2.05$ & $2.24 \pm 1.09$ & 0.035 & $1.10 \pm 0.14$ & $1.00 \pm 0.00$ & 0.03 & $1.20 \pm 0.23$ & $0.32 \pm 0.14$ & $<.001$ \\
\hline
\end{tabular}

Results are expressed as mean \pm SD. P values as compared two groups in each period.

in the non-EMG group in all periods (Table 2). Using the outof-plane approach, the effect of electromagnetic guidance (group $\times$ time interaction) was not significant regarding the time required for needle placement $(\mathrm{P}=0.119$; repeated measures ANOVA) or the number of needle advances $(\mathrm{P}=0.107$; repeated measures ANOVA); however, electromagnetic guidance significantly improved the score for the ultrasound view $(\mathrm{P}=0.029$; mixed models regression analysis). The time required for needle placement was significantly shorter in the EMG group than in the non-EMG group in all experimental periods. However, the number of needle advances did not differ significantly between groups in the $\mathrm{P} 1-\mathrm{P} 5$ periods. The score for the ultrasound view was significantly lower in the EMG group than in the non-EMG group in all periods (Table 3 ).

\section{Discussion}

In this study we compared the early learning curves of novices performing ultrasound-guided needle placement in phantom models with or without electromagnetic guidance (eZGuide ${ }^{\mathrm{TM}}$ ). Using an in-plane approach, the electromagnetic guidance significantly decreased the time required for needle placement and the number of needle advances in the P1 period (first five attempts) and $\mathrm{P} 2$ period (second five attempts); but differences between the two groups gradually decreased over time. The effect of the EMG system was significant especially in the early learning period of present study.

The number of needle advances is associated with complications [15-19], suggesting that electromagnetic guidance can reduce the risk of complications for novices performing needle placement using an in-plane approach during the very early learning period. The electromagnetic guidance may also help novices perform needle placement more quickly using the outof-plane approach, but may not decrease the risk of complications associated with multiple needle advances, or repositioning in this early learning period. The electromagnetic guidance does not decrease the need for needle repositioning using the outof-plane approach because the needle placement technique is different. For the in-plane approach with long-axis view, operators should maintain an optimal view and confirm the needle tip position whenever possible. However, using an out-of-plane approach with short-axis view, the operator does not need to confirm the needle tip position on the ultrasound view. For that reason, the non-EMG group was as successful in needle placement using the out-of-plane approach as the EMG group.

The electromagnetic guidance system also improved the ultrasound view using both in-plane and out-of-plane approaches in all experimental periods. With electromagnetic guidance novices could confirm the needle tip position, and the score for the ultrasound view decreased over time. The most common error among novices is advancing the needle without visualizing the needle tip [5], which may lead to damage of the surrounding 
tissue or organ injury [20,21]. Thus, the electromagnetic guidance may prevent complications caused by failure to confirm the needle position.

In the present study, novices not only took longer time to place the needle but also needed to reposition the needle more frequently using the in-plane approach compared with the outof-plane approach. These findings are consistent with previous studies [22,23]. Blaivas et al. [24] also reported that vascular access was obtained more rapidly using the out-of-plane approach. Similarly, Chittoodan et al. [25] reported that the success rate at first access was higher with the out-of-plane approach than the in-plane approach. These results may be explained by the difficulty in keeping the needle in view using the in-plane approach [11]. Thus, if both approaches are available, the out-ofplane approach may be more advantageous because it decreases the time required for needle placement. However, if the inplane approach is the only available option, the electromagnetic guidance can help novices decrease the time required for needle placement and confirm the position of the needle on ultrasound view.

The phantom model used in this study, which was rectangular with a $0.3-\mathrm{cm}$ diameter echogenic stick at a depth of 1.5 $\mathrm{cm}$, may have influenced our results. Vascular catheterization and nerve block are usually performed with ultrasound guidance, and we therefore chose a model with a tube-shaped target similar to vessels and nerves. However, in the present study the operator did not need to place the needle at the appropriate depth because passing through the target was impossible using this phantom model. The same phantom was used during every needle placement attempt in our study; therefore, these results should be interpreted with a caution and may not be applicable to other types of needle placement.
There are several limitations of this study. First, the use of a phantom model may underestimate the operators' fear of needle placement in a patient. Second, we did not evaluate the later learning curve of novices learning ultrasound-guided needle placement. However, the number of attempts used in this study was based on a study by Kopacz et al. [11], which reported that 25 procedures are necessary to improve techniques of regional anesthesia. Third, neither the participants nor the observers could be blinded because activation of the electromagnetic guidance system was displayed on the ultrasound screen. Fourth, the participants of the EMG group did not perform needle placement under general ultrasound guide. Therefore, whether the participants of the EMG group perform the needle placement using general ultrasound better than those of the non-EMG group is unclear.

In conclusion, medical navigation systems such as electromagnetic guidance may be beneficial when performing ultrasound guided peripheral nerve blocks or vascular cannulation in the early learning period, especially by inexperienced operators with reducing patient risk. However, clinical studies are needed to confirm this finding.

\section{Acknowledgments}

The authors thank Daehyung Lee for his helpful assistance with data analysis.

\section{ORCID}

Jeongyun Song, http://orcid.org/0000-0002-4434-4783

Hyo Jin Byon, http://orcid.org/0000-0002-5400-6155

\section{References}

1. Marhofer P, Greher M, Kapral S. Ultrasound guidance in regional anaesthesia. Br J Anaesth 2005; 94: 7-17.

2. Marhofer P, Harrop-Griffiths W, Kettner SC, Kirchmair L. Fifteen years of ultrasound guidance in regional anaesthesia: part 1. Br J Anaesth 2010; 104: 538-46.

3. Tsui B. Ultrasound-guidance and nerve stimulation: implications for the future practice of regional anesthesia. Can J Anaesth 2007; 54: 165-70.

4. Bröking K, Waurick R. How to teach regional anesthesia. Curr Opin Anaesthesiol 2006; 19: 526-30.

5. Sites BD, Spence BC, Gallagher JD, Wiley CW, Bertrand ML, Blike GT. Characterizing novice behavior associated with learning ultrasoundguided peripheral regional anesthesia. Reg Anesth Pain Med 2007; 32: 107-15.

6. Kessler J, Bolger AF, Gray AT. An essential skill. Reg Anesth Pain Med 2006; 31: 498-500.

7. Sites BD, Brull R, Chan VW, Spence BC, Gallagher J, Beach ML, et al. Artifacts and pitfall errors associated with ultrasound-guided regional anesthesia. Part I: understanding the basic principles of ultrasound physics and machine operations. Reg Anesth Pain Med 2007; 32: 412-8.

8. Sites BD, Brull R, Chan VW, Spence BC, Gallagher J, Beach ML, et al. Artifacts and pitfall errors associated with ultrasound-guided regional anesthesia. Part II: a pictorial approach to understanding and avoidance. Reg Anesth Pain Med 2007; 32: 419-33.

9. de Oliveira Filho GR, Helayel PE, da Conceição DB, Garzel IS, Pavei P, Ceccon MS. Learning curves and mathematical models for interventional ultrasound basic skills. Anesth Analg 2008; 106: 568-73.

10. Randomised trial to assess safety and fetal outcome of early and midtrimester amniocentesis. The Canadian Early and Mid-trimester 
Amniocentesis Trial (CEMAT) Group. Lancet 1998; 351: 242-7.

11. Kopacz DJ, Neal JM, Pollock JE. The regional anesthesia "learning curve". What is the minimum number of epidural and spinal blocks to reach consistency? Reg Anesth 1996; 21: 182-90.

12. Edwards L. Applied analysis of variance in behavioral science. New York, CRC Press. 1993, pp 105-10.

13. Muller KE, Barton CN. Approximate power for repeated-measures ANOVA lacking sphericity. J Am Stat Assoc 1989; 84: 549-55.

14. Muller KE, Lavange LM, Ramey SL, Ramey CT. Power calculations for general linear multivariate models including repeated measures applications. J Am Stat Assoc 1989; 84: 549-55.

15. Randolph AG, Cook DJ, Gonzales CA, Pribble CG. Ultrasound guidance for placement of central venous catheters: a meta-analysis of the literature. Crit Care Med 1996; 24: 2053-8.

16. Teichgräber UK, Benter T, Gebel M, Manns MP. A sonographically guided technique for central venous access. AJR Am J Roentgenol 1997; 169: 731-3.

17. Hadzic A, Sala-Blanch X, Xu D. Ultrasound guidance may reduce but not eliminate complications of peripheral nerve blocks. Anesthesiology 2008; 108: 557-8.

18. Froehlich CD, Rigby MR, Rosenberg ES, Li R, Roerig PL, Easley KA, et al. Ultrasound-guided central venous catheter placement decreases complications and decreases placement attempts compared with the landmark technique in patients in a pediatric intensive care unit. Crit Care Med 2009; 37: 1090-6.

19. Mansfield PF, Hohn DC, Fornage BD, Gregurich MA, Ota DM. Complications and failures of subclavian-vein catheterization. N Engl J Med 1994; 331: 1735-8.

20. Berk D, Gurkan Y, Kus A, Ulugol H, Solak M, Toker K. Ultrasound-guided radial arterial cannulation: long axis/in-plane versus short axis/ out-of-plane approaches? J Clin Monit Comput 2013; 27: 319-24.

21. Ruiz A, Sala-Blanch X, Martinez-Ocón J, Carretero MJ, Sánchez-Etayo G, Hadzic A. Incidence of intraneural needle insertion in ultrasoundguided femoral nerve block: A comparison between the out-of-plane versus the in-plane approaches. Rev Esp Anestesiol Reanim 2014; 61: 73-7.

22. Tielens LK, Damen RB, Lerou JG, Scheffer GJ, Bruhn J. Ultrasound-guided needle handling using a guidance positioning system in a phantom. Anaesthesia 2014; 69: 24-31.

23. Whittaker S, Lethbridge G, Kim C, Keon Cohen Z, Ng I. An ultrasound needle insertion guide in a porcine phantom model. Anaesthesia 2013; 68: 826-9.

24. Blaivas M, Brannam L, Fernandez E. Short-axis versus long-axis approaches for teaching ultrasound-guided vascular access on a new inanimate model. Acad Emerg Med 2003; 10: 1307-11.

25. Chittoodan S, Breen D, O’Donnell BD, Iohom G. Long versus short axis ultrasound guided approach for internal jugular vein cannulation: a prospective randomised controlled trial. Med Ultrason 2011; 13:21-5. 